\title{
Study on the ions' behavior in an electron cyclotron resonance plasma
}

\author{
Minghai $\mathrm{Liu}^{\text {a) }}$ and Xiwei $\mathrm{Hu}^{\mathrm{b})}$ \\ Department of Modern Physics,University of Science and Technology of China, Hefei, Anhui 230026, \\ People's Republic of China \\ Hanming Wu \\ Institute of Mechanics, Academia Sinica, Beijing 100080, People's Republic of China
}

(Received 14 October 1996; accepted for publication 28 February 1997)

\begin{abstract}
The energy, velocity, angle distribution of ions in magnetoactive electron cyclotron resonance plasma have been studied with a two-dimension hybrid mode. The dependence of these distribution functions versus position and pressure are discussed. Our simulation results are in good agreement with many experimental measurements. (C) 1997 American Institute of Physics.
\end{abstract}

[S0021-8979(97)05411-X]

\section{INTRODUCTION}

The application of electron cyclotron resonance (ECR) plasma has received a great deal of attention in the microelectronic fabrication for many years. ${ }^{1,2}$ Recently, one of the most important issues is the behavior of ions which is directly related to the performance of plasma processing such as dry etching, sputtering and film deposition, etc. For this reason, ion energy, velocity distribution functions and temperature have been investigated in detail experimentally. ${ }^{3-11}$ For example, Sadghi et al. ${ }^{3}$ reported a detailed measurement of ion velocity distributions in both the source and reactor region in an ECR system. King and co-workers ${ }^{5}$ have investigated the ion energy distribution in two magnetic field configurations. These measurements are necessary for understanding the evolution and uniformity of ion energy and velocity distribution functions (IEDF and IVDF) from source to downstream and how these functions are influenced by their dependence on the plasma parameters such as pressure, power, position, magnetic field, etc. However, the correspondent theoretical and numerical research are very scarce in these aspects. Uhm and his collaborators ${ }^{12}$ pointed out theoretically that the full width of half-maximum (FWHM) of IEDF is a decreasing function of the chamber pressure. Porteous ${ }^{13}$ and colleagues ${ }^{14,15}$ simulated the variation of ion energy and impact angle distribution with radial positions, but only at the bottom (substrate) surface. In this paper, we follow the latter physical model to study the ions' energy, velocity and angle distribution in the whole downstream. The goal of this work is to understand the ions' transport properties from upstream to the substrate surface in the chamber. We provide a brief review of the design features of the ECR reactor and the physical model in Sec. II, followed by a discussion of our simulation results in Sec. III. The conclusions are in Sec. IV.

\section{THE DESIGN FEATURES AND PHYSICAL MODEL}

\section{A. The design features of the ECR reactor}

A description of the ECR reactor has been given previously. ${ }^{14,15}$ The schematic diagram of the ECR system

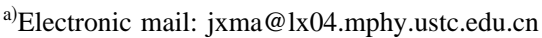

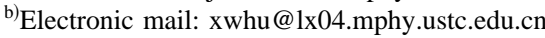

and magnetic field profile are shown in Figs. 1(a) and 1(b). An $850 \mathrm{~W}$ microwave $(2.45 \mathrm{GHz})$ was supplied to the vacuum chamber $(32 \mathrm{~cm}$ in diameter, $22 \mathrm{~cm}$ in length) through a quartz window $(24 \mathrm{~cm}$ in diameter). For a compact ECR reactor(the ratio of the source length to the chamber's is $1: 4)$, the resonant zone sites are on the top of the downstream whose axial position is about $9-10 \mathrm{~cm}$. The reactor walls are grounded except for the microwave dielectric window. Argon gas is filled at 5 mTorr. The divergent magnetic field was established by two external coils.

\section{B. Physical model}

The characteristics of the physical model used in this paper have been described in detail elsewhere ${ }^{13-15}$, so the following description is quite brief.

\section{Electron description}

Electron is modeled as a fluid with mass conservation

$$
\begin{aligned}
& \mathbf{j}_{\mathbf{e}}=\mu\left[n_{e} \nabla \phi-\nabla\left(n_{e} T_{e}\right) / e\right], \\
& \nabla \cdot \mathbf{j}_{\mathbf{e}}=R_{\text {ion }}\left(n_{e}, n_{i}, n_{0}\right) ;
\end{aligned}
$$

energy conservation

$$
\begin{aligned}
& \mathbf{Q}=2 \kappa T_{e}\left(\mathbf{j}_{e}-\mu n_{e} \nabla T_{e} / e\right), \\
& \nabla \cdot \mathbf{Q}=P_{e c r}+e \mathbf{j}_{\mathbf{e}} \cdot \nabla \phi-P_{\text {loss }} ;
\end{aligned}
$$

and Poisson equation

$$
\epsilon_{0} \Delta \phi=e\left(n_{e}-n_{i}\right),
$$

where $n_{e}, n_{i}, n_{0}, \phi, T_{e}, \mathbf{j}_{e}, \mathbf{Q}, \mu, R_{i o n}\left(n_{e}, n_{i}, n_{0}\right)$, and $\varepsilon_{0}$ are electron density, ion density, neutral density, electrostatic potential, electron temperature, electron flux, electron energy flux, mobility, ionization rate and permeability, respectively. $P_{\text {loss }}=P_{I}+P_{E X}+P_{M E}+P_{E L}$, where $P_{I}, P_{E X}$, $P_{M E}, P_{E L}$ are the power consumption for ionization, excitation, metastable excitation and elastic-neutral collision, respectively. Their detail expressions can be found in Ref. 13 . $P_{e c r}$ is the power input into electrons from microwave field, its self-consistent treatment would involve a full wave numerical solution with proper boundary condition. This is not our main issue in the present paper, so we just take it from experience as a formula such as in Ref. 13: $P_{e c r}$ $=A\left[1.2-(r / R)^{4}\right] /\left(1+\left\{\left[B(r, z)-B_{0}\right] /(\Delta B)\right\}^{2}\right)$, where $R$ is 

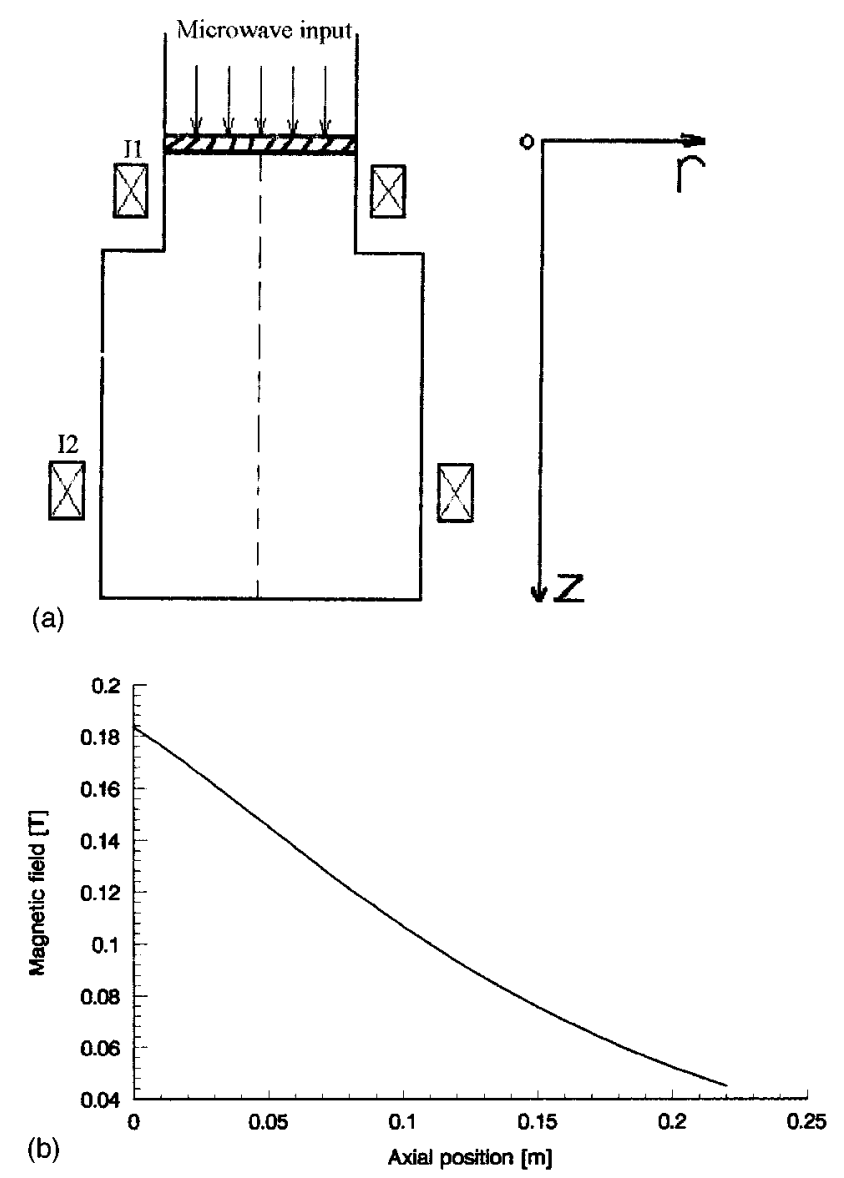

FIG. 1. Schematic diagram of the ECR system and magnetic field profile.

the chamber radius, $A$ is constant determined with normalization of total power input and $B_{0}=950 \mathrm{G}$ is the resonance magnetic field, and $\Delta B$ is $25 \mathrm{G}$.

\section{Ion description}

Ions are considered as individual particles and their dynamic behaviors are described by combining a Partical-incell and Monte Carlo code in two spatial dimensions $(r, z)$ and three velocity space dimensions $\left(v_{r}, v_{\theta}, v_{z}\right)$ when they move into the applied magnetic field and self-consistent electrostatic field. Ions are generated by the ionization or charge exchange event. Ions created from the ionization event will have the background gas temperature and corresponding Maxwellian velocity distribution. Ions generated from the ion-neutral collisions (only the charge exchange collision is considered) will possess the temperature and random thermal velocity of the neutral atom from which they were created. Fast neutrals created from charge exchange are not followed further. Ion-ion and ion-electron collisions are ignored.

\section{Boundary conditions}

The sheath is assumed to be infinitesimally thin. The potential discontinuity between plasma sheath and reactor wall is defined as $\Delta \phi=\phi_{s}-\phi_{w}$. The magnitude of the sheath potential jump discontinuity determines the magnitudes of electron flux, electron energy flux and ion energy impacted onto the wall. For collisionless sheath, the three

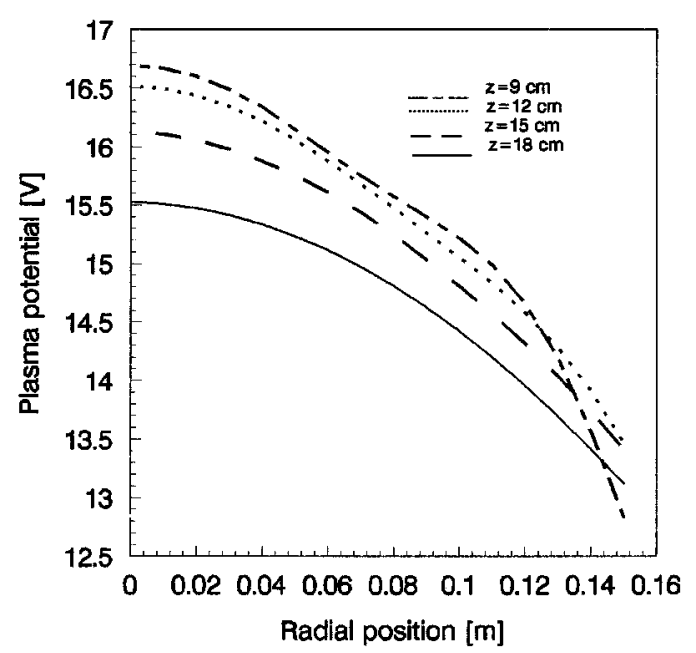

FIG. 2. The variation of plasma potential with radial position at different axial positions: power $=850 \mathrm{~W}$, pressure $=5$ mTorr.

aspects mentioned depend on neither the sheath thickness nor the shape of the sheath potential profile, but only on the magnitude of $\Delta \phi$. In a Maxwellian electron velocity distribution, the magnitude of the electron flux to the wall is

$$
j_{e w}=\frac{1}{4} n_{e s} v_{e s} \exp \left[(-e \Delta \phi) /\left(\kappa T_{e s}\right)\right],
$$

where $T_{e s}, n_{e s}$ and $v_{e s}$ are electron temperature, electron density, and average thermo-velocity at the plasma-sheath boundary, respectively. The electron energy flux is represented analogously by

$$
Q_{e w}=\kappa T_{e s} j_{e w}\left[2+(e \Delta \phi) /\left(\kappa T_{e s}\right)\right] .
$$

The second kind of boundary is of a dielectric wall, where the surface potential will float with respect to ground. The wall potential $\phi_{w}$ is determined by the ion flux $j_{i w}$ $\left(=j_{\text {ew }}\right)$ at each grid point of boundary as follows:

$$
\phi_{w}=\phi_{s}+\kappa T_{e} / e \ln \left[4 j_{i w} /\left(n_{e s} v_{t h}\right)\right] .
$$

\section{RESULTS AND DISCUSSION}

Many important plasma parameters can be obtained from this simulation, such as plasma density, electron temperature, plasma potential, ion current density into the wall, etc. In this paper, the ion properties are our object of interest.

The variations of plasma potential with radial position at different axial positions are given in Fig. 2. The plasma potential decreases with the increasing radial position at each axial position. In addition, the radial electrostatic field decreases with increasing axial position. The dependence of ion density versus radial position at different axial positions is shown in Fig. 3. The ion density decreases as the radial position increases for a given position $z$, and the axial density gradient has an ascending negative value along the axial position. These results are useful to interpret the following ions' performance. 


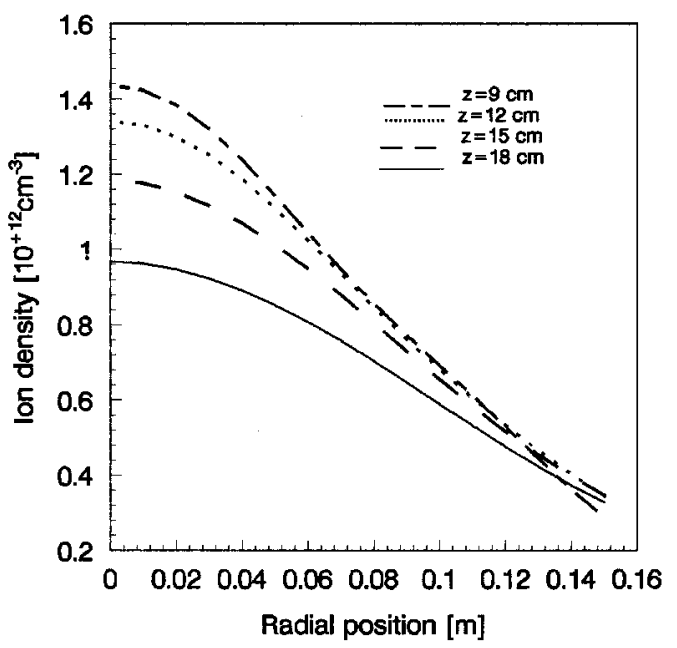

FIG. 3. The dependence of ion density vs radial position at different axial positions. The conditions are as shown in Fig. 1.

\section{A. Ion energy distribution versus pressure at substrate surface}

Figure 4 shows the dependence of ion energy distribution versus pressure at substrate surface. As usual, the IEDF can be characterized by three parameters: the average energy, the probable energy and the FWHM energy. It can be seen that all three parameters increase with decreasing pressure. These trends are consistent with Uhm's theoretical report $^{12}$ and experimental measurement. ${ }^{5-8}$

When an ion injects into the substrate, its impact angle is generally not perpendicular to the surface. Defining the ion impact radial pitch angle as $\operatorname{arctg}\left(v_{r} / v_{z}\right)$, the ion impact radial angle distributions for various pressures are plotted in Fig. 5. It is obvious from the graph that a lower pressure results in a smaller FWHM of the distribution, although the ion radial angles shift to higher values.

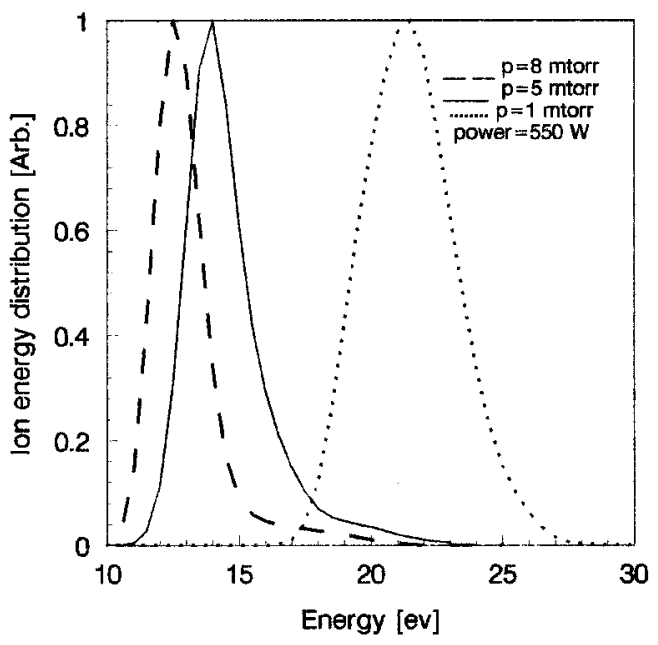

FIG. 4. The dependence of ion energy distribution versus pressure at substrate surface. $z=22 \mathrm{~cm}, 12<r<14 \mathrm{~cm}$.

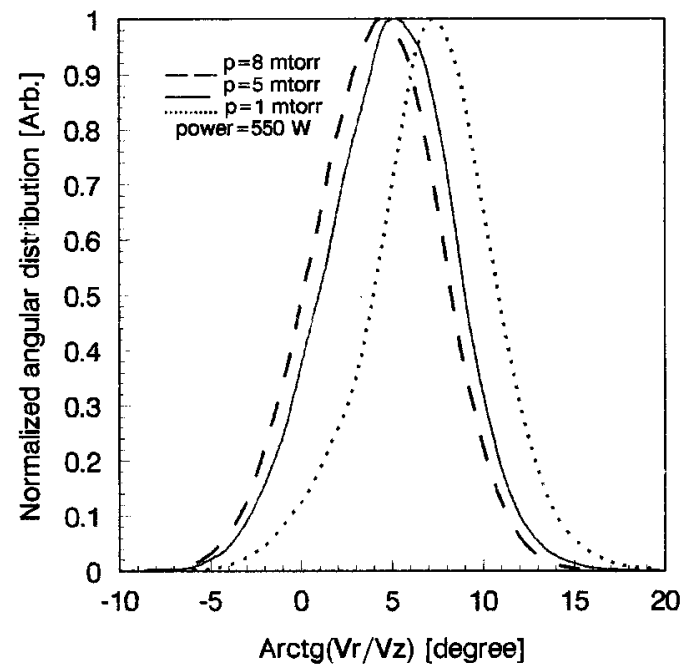

FIG. 5. The ion impact radial angle distributions for various pressure at substrate surface. The positions are as given in Fig. 4.

\section{B. Ion velocity distribution versus position $z$ in the downstream}

In order to study the ion energy in more detail, the ion velocity distributions at different downstream positions were investigated. We select five different grids $(1 \times 1 \mathrm{~cm})$ along the axial midplane $(0<r<1 \mathrm{~cm})$ to collect and analyse statistically the ions information. Figure 6 shows the axial velocity distribution at different positions in the downstream. As position $z$ increases, $f\left(v_{z}\right)$ shifts to higher velocity due to the acceleration along an axial plasma electrostatic field, and the FWHMs of the distributions increase with the axial positions.

Another attractive feature of $f\left(v_{z}\right)$ is that their left (negative velocity) sides have almost no change along the axial position. This could be understood as the ions are created from either ionization or charge exchange events in our model, the velocities of new born ions are very slow and

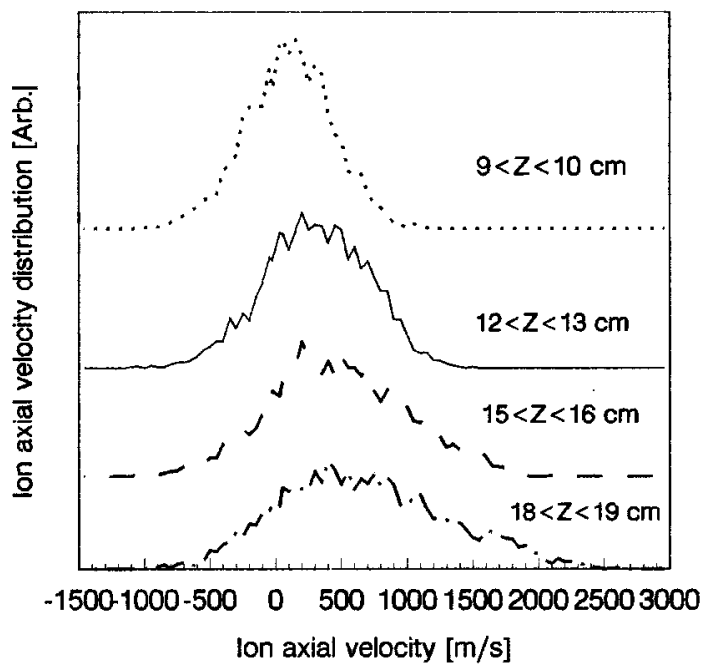

FIG. 6. The $z$-velocity distribution at different axial position $(0<r<1 \mathrm{~cm})$ in the downstream. The conditions are as shown in Fig. 2. 


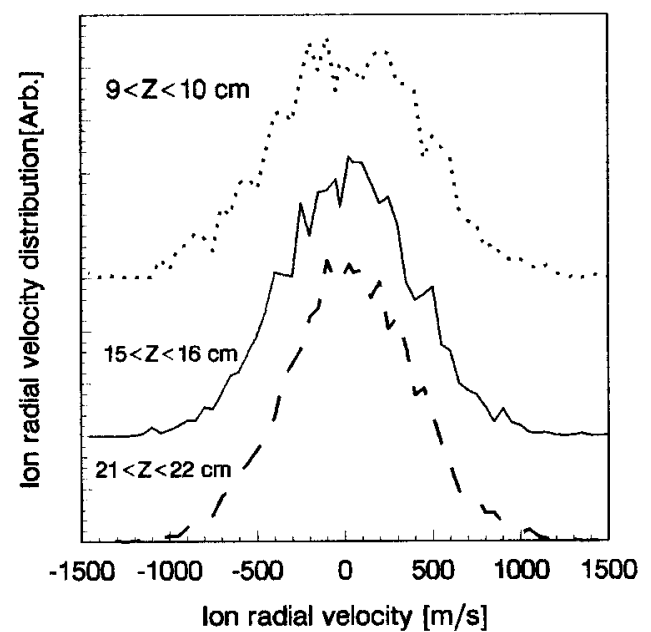

FIG. 7. The $r$-velocity distribution at different axial position $(0<r<1 \mathrm{~cm})$ in the downstream. The conditions are as given in Fig. 2.

random. At each time step of collecting the ions information, these new born ions still keep the new born distribution and don't undergo the electrostatic field acceleration, so the left sides of distribution remain unchanged. This result is qualitatively in good agreement with those reported by King et $a .^{5}$ using LIF technique at small $z$ values under similar operating conditions.

The dependence of radial and azimuthal velocity distributions on the axial positions are given in Figs. 7 and 8, respectively. Both the average velocity and the FWHM of each distribution decrease with increasing axial positions. As shown in Figs. 2 and 3, the radial electrostatic field $E_{r}$ and the density gradient $\nabla_{r} n_{i}$ are a decreasing function with the position $z$, and the magnetic field has the same variation trend as Fig. 1(b); meanwhile the axial electrostatic field $E_{z}$ and the density gradient $\nabla_{z} n_{i}$ have the opposite tendency. So the ion drift velocity resulted from the $\mathbf{E} \times \mathbf{B}$ and $\nabla n_{i} \times \mathbf{B}$ decrease as the axial position increases. Here we analyze quantitatively the azimuthal velocity of ions in detail. In the

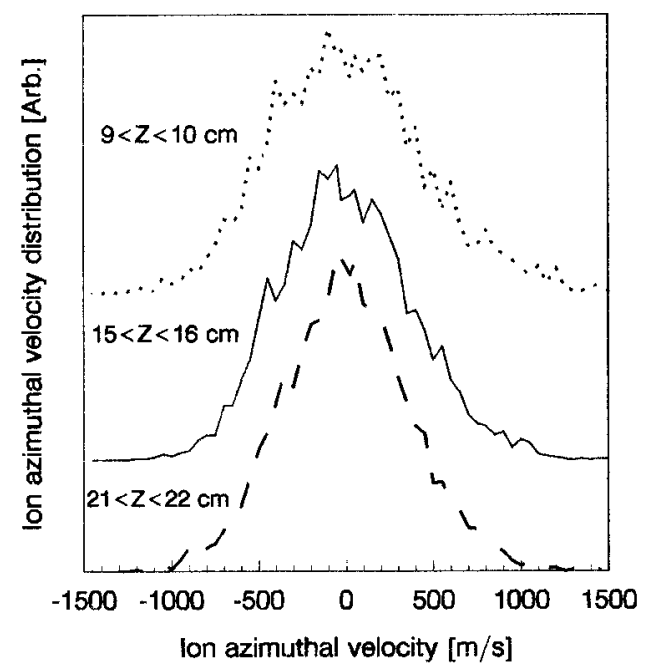

FIG. 8. The $\theta$-velocity distribution at different axial positions $(0<r<1 \mathrm{~cm})$ in the downstream. The conditions are as shown in Fig. 2. existence of the electrostatic field and density gradient, the ion rotation velocity around $\mathbf{B}$ can be given by: ${ }^{16}$

$$
v_{\theta}=\mathbf{E} \times \mathbf{B} / B^{2}-\nabla p \times \mathbf{B} / e n_{i} B^{2}=v_{e}+v_{d},
$$

where $\nabla p=\kappa T \nabla n_{i}$ represents the effect of ion pressure gradient, and $v_{e}, v_{d}$ are the $\mathbf{E} \times \mathbf{B}$ drift, the diamagnetic drift induced by the ion density gradient respectively. According to the mentioned formula and using the data in Figs. 2 and 3, the theoretical average rotation velocities $v_{\theta}$ are 20.8, 19.8, 19.1 and $16.1 \mathrm{~m} / \mathrm{s}$ at axial positions of $9.5,12.5,15.5,18.5$ $\mathrm{cm}$, respectively. In our simulation, the ion azimuthal velocities calculated from the IVDFs are 25.25, 12.66, 4.867,3.87 $\mathrm{m} / \mathrm{s}$ corresponding to the four regions in Fig. 6. So the simulation results are consistent qualitatively with theoretical calculations. The discrepancies between them are caused by the following factors:

(1) The $v_{\theta}$ doesn't represent completely in line the ion azimuthal velocity because the ions don't confine well by the magnetic field as electrons, especially in the case of weak field.

(2) The effect of the ion-neutral collisions are ignored in the theoretical description.

Another important and interesting result is that the IVDF is nearly isotropic in the top of the downstream (close to resonant zone), compared with Figs. 6, 7, and 8, but as the $z$ increases, the IVDF becomes anisotropic. Note that the type of our ECR reactor is a compact one, its resonant region sites on the top of the downstream (about $9-10 \mathrm{~cm}$ in the axial position). The ionization rate and the ion density have their maximum at this region, so the high frequency of ionneutral collisions tends to randomize the ion energy, so the ion velocity is isotropic. As the axial position increases, the $z$ direction distributed plasma potential will accelerate the ions, and at the same time, both the rate of charge-exchange collisions and ionizations decrease rapidly, so the IVDF becomes more and more anisotropic.

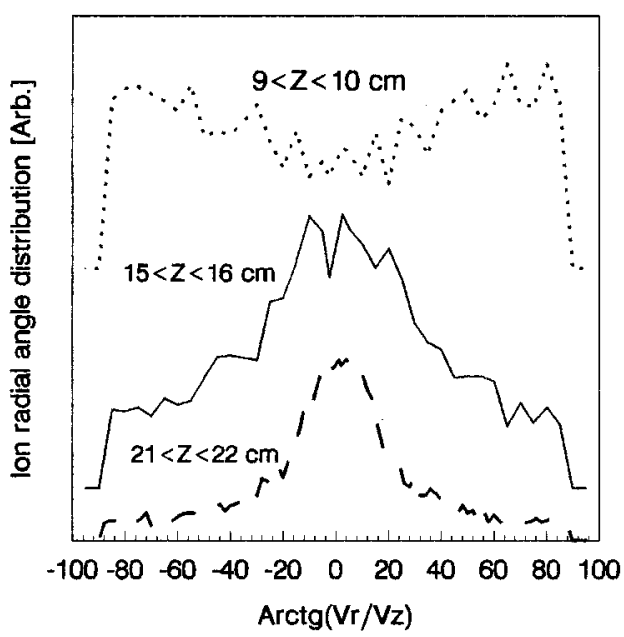

FIG. 9. The dependence of radial angle distribution on the axial position $(0<r<1 \mathrm{~cm})$. The conditions are as given in Fig. 2. 


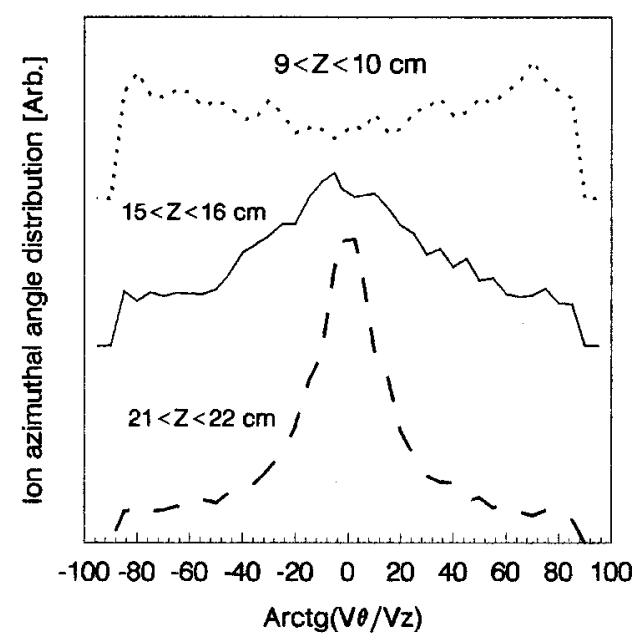

FIG. 10. The dependence of azimuthal angle distribution on the axial position $(0<r<1 \mathrm{~cm})$. The conditions are as shown in Fig. 2.

\section{Ion angle distribution versus position $z$ in the downstream}

The ion radial and azimuthal pitch angle $\left[\operatorname{arctg}\left(v_{r} / v_{z}\right), \operatorname{arctg}\left(v_{\theta} / v_{z}\right)\right]$ distributions versus position $z$ in the downstream are shown in Figs. 9 and 10, respectively. On the top of the downstream (close to resonant region), both the radial and azimuthal angle are diffusive. This can be considered as another argument of isotropic IVDF. As the axial position increases, the proportions of small angle become dominant. It can be deduced that the ion motion will converge in the axial direction in transportation from upstream to the downstream.

\section{CONCLUSION}

We have presented the study of the ions' energy, velocity and angle distribution as a function of position and pressure in the whole downstream. The main results are given in the following five aspects:

(1) The FWHM of the ion energy and angle distribution on the substrate surface is a decreasing function of the neutral pressure.

(2) The $z$-directed velocity distributions is a broadening distribution along the axial position, but their left sides of the distribution remain unchanged.
(3) The average velocity and the FWHM of $r$-directed and $\theta$-directed velocity distributions decrease with increasing downstream positions.

(4) At the resonant regions, the IVDF is nearly isotropic. As the downstream position increases, the IVDF becomes anisotropic.

(5) In the resonant zone, both the radial and azimuthal angle are diffusive. As the axial position increases, the proportion of small angle becomes dominant. This means the ion motion will converge to the axial direction in transportation from upstream to the downstream.

\section{ACKNOWLEDGMENT}

This work was supported partly by the Grant No. LWTZ-1298 of the Chinese Academy of Science and the National Nature Science Foundation of China. One of the authors thanks Dr. F. Q. Shao from Institute of Physics, Academia Sinica for his friendly communication. The encouragement and useful advice of Professor R. J. Zhan are gratefully acknowledged. Appreciation is also expressed to Dr. H. T. Hu, Professor Z. F. Ding and Q. C. Wu from the Institute of Plasma Physics, Academia Sinica for their helpful and enlightening discussions.

${ }^{1}$ J. Asmussen, J. Vac. Sci. Technol. A 7, 883 (1989).

${ }^{2}$ Z. Y. Ning and Z. X. Ren, Progress in Phys. 12, 38 (1992) (in Chinese).

${ }^{3}$ N. Sadeghi, T. Nakano, D. J. Trevor, and R. A. Gottscho, J. Appl. Phys. 70, 2552 (1991).

${ }^{4}$ D. J. Trevor, N. Sadeghi, T. Shiki, J. Derouard, R. A. Gottscho, P. D. Foo, and J. M. Cook, Appl. Phys. Lett. 57, 1188 (1990).

${ }^{5}$ G. King, F. C. Sze, P. Mak, T. A. Grotjohn, and J. Asmussen, J. Vac. Sci. Technol. A 10, 1265 (1992).

${ }^{6}$ W. M. Holber and J. Forster, J. Vac. Sci. Technol. A 8, 3720 (1990).

${ }^{7}$ M. Matsuoka and K. Ono, J. Vac. Sci. Technol. A 6, 25 (1988).

${ }^{8}$ S. Samukawa, Y. Nakagawa, and K. Ikeda, Jpn. J. Appl. Phys. 1 30, 423 (1991).

${ }^{9}$ E. A. Den Hartog, H. Persing, and R. C. Woods, Appl. Phys. Lett. 57, 661 (1990).

${ }^{10}$ T. Nakano, N. Sadeghi, and R. A. Gottscho, Appl. Phys. Lett. 58, 458 (1991).

${ }^{11}$ Y. Okuno, Y. Ohtsu, H. Fujita, W. Chen, and S. Miyake, Jpn. J. Appl. Phys. 1 32, L1698 (1993).

${ }^{12}$ H. S. Uhm, H. Y. Chang, J. H. Kim, and S. K. Song, Phys. Plasmas 2, 991 (1995).

${ }^{13}$ R. K. Porteous, H. M. W. Wu, and D. B. Graves, Plasma Sources Sci. Technol. 3, 25 (1994).

${ }^{14}$ D. B. Graves, H. M. Wu, and R. K. Porteous, Jpn. J. Appl. Phys. 1 32, 2999 (1993).

${ }^{15}$ H. M. Wu, D. B. Graves, M. Li, and Q. Wang, Chin. Phys. Lett. 11, 747 (1994).

${ }^{16}$ F. F. Chen, Introduction to Plasma Physics (Plenum, New York, 1974). 